\title{
Antidepressant Induced Hyponatremia
}

\author{
Rudraprasad Acharya \\ RMO-Cum-Clinical Tutor \\ Medical College, Kolkata
}

\section{INTRODUCTION}

Electrolyte imbalance is a very common cause as well as comorbidity of hospitalization, especially in elderly individuals. It also enhances the morbidity and mortality in this group of patients. Disorders of sodium balance are the most prevalent types of electrolyte disturbance in clinical settings. Among several etiological factors related to disturbed serum sodium concentration, iatrogenic causes are very common. Antidepressants are noteworthy contributors among the drugs causing reduced plasma sodium concentration and as these drugs are quite commonly used in elderly patients due to high prevalence of depression in this age group, here we discuss about antidepressant induced hyponatremia.

\section{HYPONATREMIA DEFINITION}

Hyponatremia is defined as a plasma $[\mathrm{Na}+]<135$ $\mathrm{mEq} / \mathrm{L}$

\section{PREVALENCE}

In a recent community study in subjects aged 55 years or more, hyponatremia was most prevalent $(7.7 \%)$ electrolyte disturbance ${ }^{1}$. While considering hospitalized patients, the prevalence shoots upto $22 \%$.

\section{CLASSIFICATION OF HYPONATREMIA BY CLINICAL CAUSE $^{2}$}

1. Syndrome of inappropriate antidiuretic hormone secretion. (SIADH), with no diuretic use, or 'possible SIADH', with diuretic use, when serum osmolality was $<280 \mathrm{mOsmol} /$ $\mathrm{kg} \mathrm{H} 2 \mathrm{O}$, urine sodium $>30 \mathrm{mM}$, and urine osmolality $>150 \mathrm{mOsmol} / \mathrm{kg} \mathrm{H} 2 \mathrm{O}$;

2. 'hypovolemia', when serum osmolality was $<280 \mathrm{mOsmol} / \mathrm{kgH} 2 \mathrm{O}$ and urine sodium $<25 \mathrm{mM}$;

3. 'out of defined range', when serum osmolality was $>280 \mathrm{mOsmol} / \mathrm{kg} \mathrm{H} 2 \mathrm{O}$ and urine sodium 25-30 mM;

4. 'other cause', i.e. the presence of hyperglycemia (defined as a serum glucose $\geq 15 \mathrm{mM}$ ).

\section{COMMON ETIOLOGIES OF HYPONATREMIA}

\section{DISEASES ASSOCIATED WITH HYPONATREMIA ${ }^{3}$}

Active heart failure

Active liver failure

Nephrotic syndrome

Pulmonary processes as infections and malignancy

Malignancy of pancreas or duodenum

Lymphoreticular malignancy

Thymoma

Psychosis

Idiopathic SIADH

Adrenal insufficiency with no sufficient therapy Diabetes mellitus or glucose $>10$ $\mathrm{mmol} / \mathrm{L}$

Chronic obstructive pulmonary disease Smoking of cigarettes

Hypertension defined as $>160 />90 \mathrm{mmHg}$

Acquired immunodeficiency syndrome

Cerebral salt wasting syndrome

\section{DRUGS ASSOCIATED WITH HYPONATREMIA ${ }^{4}$}

Antipsychotic drugs : typical and atypical Clofibrate

Antidepressants : SSRIs, SNRIs,TCAs 


\section{Antidepressant Induced Hyponatremia}

Proton pump inhibitors

Benzodiapines NSAIDs

Anti-epileptic drugs : carbamazepine,

oxcarbazepine, valproate,

Oral hypoglycemic drug : sulfonylureas,

biguanides

Anti-Parkinson drugs

Desmopressin, vasopressin, oxytocin

Rivastigmine

Ciprofloxacin, moxifloxacin,Voriconazole

Opioid analgesics, tramadol

Chemotherapeutics : cyclophosphamide,

vincristine, vinblastine

Thiazide diuretics, loop diuretics, aldosterone antagonists

Beta Blockers, ACE inhibitors, nitrates, calcium channel blockers

\section{CLINICAL PRESENTATION}

The clinical features of hyponatremia are related to the osmotic intracellular water shift leading to cerebral edema. Therefore, the symptoms are primarily neurologic, and their severity is dependent on both the magnitude of the fall in plasma $[\mathrm{Na}+]$ and the rapidity of the decrease. In acute hyponatremia (i.e., developing in $<2$ days), patients may complain of nausea and malaise with $[\mathrm{Na}+] \sim 125 \mathrm{mEq} / \mathrm{L}$. As the plasma $[\mathrm{Na}+]$ falls further, symptoms may progress to include headache, lethargy, confusion, and obtundation. Stupor, seizures, and coma do not usually occur unless the plasma $[\mathrm{Na}+]$ falls acutely below $115 \mathrm{mEq} / \mathrm{L}$. In chronic hyponatremia (>3 days' duration), adaptive mechanisms designed to defend cell volume occur and tend to minimize the increase in ICF volume and its symptoms.

\section{ANTIDEPRESSANT HYPONATREMIA ASSOCIATION}

Prevalence and risk factors of hyponatremia as Adverse Reaction to Antidepressants (AR-AD) :

In one recent study involving elderly patients aged 60 yrs or more, who are using antidepressants, prevalence of hyponatremia as an AR-AD was $9.3 \%$. For TCA users this prevalence was $11.5 \%$, for SSRIs $10.2 \%$, for venlafaxine $8.6 \%$ and for mirtazapine 5.6\%. Risk factors were a history of hyponatremia (adjusted Odds Ratio11.17), weight $<60 \mathrm{~kg}$ (adjusted OR 3.47), and psychosis (adjusted OR 3.62). Hyponatremia was not associated with gender, age, class of antidepressant, or duration of antidepressant use according to this study ${ }^{3}$.

In another study ${ }^{5}$ involving patients aged $>18$ yrs admitted in hospital with primary or secondary diagnosis of hyponatremia, compared with nonrecipients of antidepressants the adjusted OR for hyponatraemia associated with current use of antidepressants was 3.96 for serotonergic antidepressants and 1.87 for non-serotonergic antidepressants. The overall adjusted OR for antidepressant use was 2.75 compared with nonuse of antidepressants. This study also concludes that the risk for hyponatraemia after initiation of a serotonergic antidepressant is highest during the first 2 weeks of drug therapy, although it can range from 4 days to 28 days (average 12.5 days).

Another recent review ${ }^{6}$ concluded that as far the risk of hyponatremia is concerned, odds ratios for SSRIs (1.5-21.6) were consistently higher than for TCAs (1.1-4.9). The risks associated with monoamine oxidase inhibitors, reboxetine and bupropion could not be established owing to insufficient information. Patient risk factors included older age (odds ratios 6.3) and concomitant use of (thiazide) diuretics (odds ratios 11.2-13.5).

As published in WHO drug information?, The Australian Adverse Reactions Advisory Committee (ADRAC) received a total of 311 reports of hyponatraemia involving SSRIs and venlafaxine. As a group, the SSRIs account for about one-quarter of all reports of hyponatraemia received by ADRAC, and are second only to diuretics as the group most commonly associated with hyponatraemia. In more than two-thirds of the 311 reports, the SSRI was the only suspected drug and three quarters of the reports involved females, implicating female sex to be an at risk group. A small proportion (14\%) identified concurrent use of a diuretic. The median patient age was 77 years (range 13 to 99 ); about $85 \%$ 


\section{Antidepressant Induced Hyponatremia}

were older than 60 years. Onset was usually within the first month of treatment. In about two-thirds of cases, full recovery followed withdrawal of the SSRI and fluid restriction. Three cases had a fatal outcome related to hyponatraemia.

The largest review of reported cases of hyponatremia ${ }^{8}$ involved 736 cases of hyponatremia and SIADH associated with SSRI use. Fluoxetine was involved in $554(75.3 \%)$ of the cases, paroxetine in $91(12.4 \%)$, sertraline in $86(11.7 \%)$ and fluvoxamine in $11(1.5 \%)$. Median time to onset of hyponatremia was 13 days (range 3 to 120 days).

\section{MECHANISM OF HYPONATREMIA BY ANTIDEPRESSANTS}

There are three possible ways drugs can affect water homeostasis:

- They can increase ADH secretion centrally

- Potentiate the effect of endogenous $\mathrm{ADH}$ at the renal medulla

- Reset the osmostat, thus lowering the threshold for ADH secretion.

ADH secretion from the posterior lobe of the pituitary gland is thought to be affected by such nonosmolar factors as angiotensin II, noradrenaline, acetylcholine and endorphin levels. Dopamine antagonists such as haloperidol and domperidone have been shown in animal models to facilitate $\mathrm{ADH}$ secretion and increase thirst by increasing angiotensin II levels. Noradrenaline induces the release of $\mathrm{ADH}$ via alpha1 adrenergic receptors; stimulation of alpha2adrenergic and beta-adrenergic receptors may, however, inhibit ADH release. Serotonin-mediated effects on 5-HT2 and 5-HT1C receptors have been shown to induce release of ADH. Although the SSRIs are believed to be highly selective in their effect on serotonin reuptake, animal studies have shown that the SSRIs have some inhibitory effects on the reuptake of dopamine and noradrenaline. As a result, antidepressants causes a syndrome of inappropriate antidiuretic hormone (ADH) secretion (SIADH) leading to increased water retention and hyponatremia.
Hyponatremia associated with SSRI use may be a manifestation of a drug interaction. SSRIs are known to inhibit a number of cytochrome P450 isoenzymes 9 . In several of the cases the patients concurrently receive a drug known to cause SIADH. By inhibiting the metabolism of concomitant drugs such as neuroleptics, adverse effects on sodium metabolism may be augmented.

\section{CLINICAL RELEVANCE}

SSRIs are usually considered safer than older antidepressant agents and therefore are often recommended as first-choice drugs in the treatment of depression and anxiety disorders. However, the documented relatively higher hyponatremia risk profiles of SSRIs imply that this recommendation might not necessarily be appropriate for specific patients. As the potential for specific adverse events varies for the different antidepressant classes, class-or drug-specific side-effect profiles should be considered together with patient-specific risk factors when deciding on the optimal treatment for individual patients. Clinicians' awareness of the higher risk and signs of hyponatremia associated with certain antidepressants should be raised, as particularly in psychiatric populations, clinical symptoms of hyponatremia can be misinterpreted as a worsening of the primary illness. Sodium levels should be checked in all elderly patients exhibiting abrupt or unexplained changes in mental status (e.g., lethargy or confusion) at any time during treatment with an antidepressant, especially SSRI or venlafaxine. Some authors recommend a routine serum sodium analysis within the first 2 weeks after initiating such treatment in patients with additional risk factors for hyponatremia, such as older age, female sex, diuretic use, low BMI, and a baseline plasma sodium level $<138 \mathrm{mmol} / \mathrm{L}^{10,11,12}$. A thorough cost-benefit analysis seems warranted to validate the latter recommendation. 


\section{Antidepressant Induced Hyponatremia}

\section{TREATMENT $^{13}$}

Management requires one to determine :

a The rate of correction

- The appropriate intervention

$\checkmark \quad$ The presence of other underlying disorders

\section{ACUTE SYMPTOMATIC HYPONATREMIA}

Severe symptomatic hyponatremia, with evidence of neurologic dysfunction, should generally be treated promptly with hypertonic saline. The risks of correcting hyponatremia too rapidly are volume overload and the development of central pontine myelinolysis (CPM). CPM is thought to result from damage to neurons resulting from rapid osmotic shifts. In its most overt form, it is characterized by flaccid paralysis, dysarthria, and dysphagia, and in more subtle presentations, it can be confirmed by computed tomography (CT) scan or magnetic resonance imaging (MRI) of the brain. The risk of precipitating CPM is increased with correction of the $[\mathrm{Na}+]$ by $>12 \mathrm{mEq} / \mathrm{L}$ in a 24-hour period.In addition to overaggressive correction, other risk factors for developing CPM include preexisting hypokalemia, malnutrition, and alcoholism.

\section{MEDICATIONS}

- Loop diuretics promote urinary excretion of water by reducing the concentration gradient necessary to reabsorb water in the distal nephron and impair the ability to excrete concentrated urine.

- Lithium and demeclocycline interfere with the collecting tubule's ability to respond to ADH, but are rarely used because of significant side effects. They should only be considered in severe hyponatremia that is unresponsive to more conservative measures.

- Vasopressin antagonists promote a water diuresis and may be useful in the therapy of SIADH. As of yet, the only formulation approved in the United States is conivaptan, which is an IV preparation and thus not applicable to chronic outpatient management of euvolemic hyponatremia.

\section{LIFESTYLE/RISK MODIFICATION}

- Oral fluid intake should be less than daily urine output.

- SIADH should first be distinguished from the conditions, which stimulate vasopressin secretion. The standard first-line therapy is water restriction and correction of any contributing factors (nausea, pneumonia, drugs, etc.).

- Water restriction. The amount of fluid restriction necessary depends on the extent of water elimination. A useful guide to the necessary degree of fluid restriction is as follows:

$\diamond \quad$ If (Urine $\mathrm{Na}+$ Urine $\mathrm{K}$ )/Serum $\mathrm{Na}<0.5$, restrict to $1 \mathrm{~L} / \mathrm{d}$.

$\diamond \quad$ If (Urine $\mathrm{Na}+$ Urine $\mathrm{K}$ )/Serum $\mathrm{Na}$ is 0.5 to 1.0 , restrict to $500 \mathrm{~mL} / \mathrm{d}$.

\section{CONCLUSION}

Hyponatremia is a potentially dangerous and hazardous side effect of antidepressants, which at instances can be proved fatal. Though it's most commonly associated with SSRIs, but other antidepressants can also cause it in fare instances. The risk of developing it is mainly during the first few weeks of drug treatment and in certain high risk groups such as elderly females. Physicians should be aware of this side effect, should be able to recognize the symptoms and if need arises, should be able to manage it properly.

\section{REFERENCES}

1. Liamis G, Rodenburg EM, Hofman A, et al. Electrolyte disorders in community subjects : prevalence and risk factors. Am J Med 2013; 126 (3) : 256-63.

2. Milionis H, Liamis G, Elisaf M. The hyponatremic patientw : a systematic approach to laboratory diagnosis. CMAJ 2002; 166 (8) : 1056-62.

3. Mannesse CK, Jansen PAF, Van Marum RJ, et al. Characteristics, prevalence, risk factors, and underlying mechanism of hyponatremia in elderly patients treated with antidepressants : A cross-sectional study. Maturitas 2013; 76: 357-63. 


\section{Antidepressant Induced Hyponatremia}

4. Mannesse CK, Vondeling AM, van Marum RJ, et al. Prevalence of hyponatremia on geriatric wards compared to other settings over four decades: A systematic review. Ageing Research Reviews 2013; 12 : 165-73.

5. Movig KLL, Leufkens HGM, Lenderink AW, et al. Serotonergic antidepressants associated with an increased risk for hyponatraemia in the elderly. Eur J Clin Pharmacol 2002; 58 : 143-148.

6. DePicker L, DenEede FV, Dumont G, et al. Antidepressants and the Risk of Hyponatremia : A Class-by-Class Review of Literature. Psychosomatics 2014; 55 : 536-47.

7. WHO Drug Information 2003; 17(3) : 150.

8. Liu BA, Mittmann N, Knowles SR, et al. Hyponatremia and the syndrome of inappropriate secretion of antidiuretic hormone associated with the use of selective serotonin reuptake inhibitors : a review of spontaneous reports. Can Med Assoc J 1996; 155 (5) : 519-27.

9. Crewe JK, Lennard MS, Tucker GT, et al. The effect of selective serotonin re-uptake inhibitors on cytochrome
P4502D6 (CYP2D6) activity in human liver microsomes. Br J Clin Pharmacol 1992; 347 : 262-65.

10. Degner D, Grohmann R, Kropp S,et al. Severe adverse drug reactions of antidepressants: results of the German multicenter drug surveillance program AMSP. Pharmacopsychiatry 2004; 37 : S39-S45.

11. Fabian TJ, Amico JA, Kroboth PD, et al. Paroxetine induced hyponatremia in older adults : a 12 week prospective study. Arch Intern Med 2004; 164(3) : 327-32.

12. Bouman WP, Pinner G, Johnson H. Incidence of selective serotonin reuptake inhibitor (SSRI)induced hyponatraemia due to the syndrome of inappropriate antidiuretic hormone (SIADH) secretion in the elderly. Int J Geriatr Psychiatry 1998; 13(1) : 12-15.

13. Sankarpandian B, Cheng S (2010). Fluid and Electrolyte Management; The Washington Manual ${ }^{\circledR}$ of Medical Therapeutics (33rd edn); 2010; Lippincott Williams \& Wilkins; Philadelphia; 375-81. 